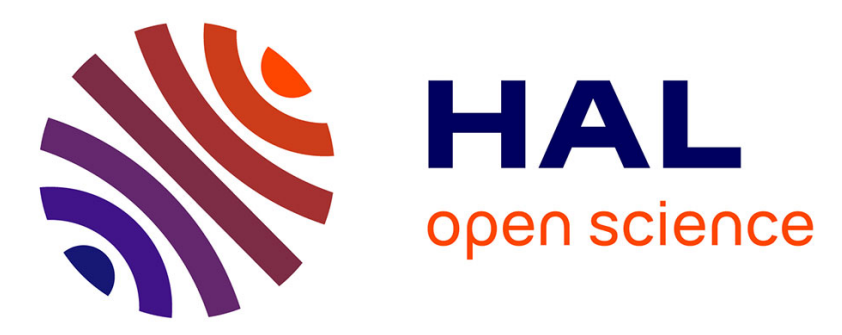

\title{
Utilisation d'ordinateurs dans l'analyse des spectres de molécules diatomiques
}

Bernard Guerin, Jean d'Incan

\section{To cite this version:}

Bernard Guerin, Jean d'Incan. Utilisation d'ordinateurs dans l'analyse des spectres de molécules diatomiques. Revue de Physique Appliquée, 1966, 1 (3), pp.239-242. 10.1051/rphysap:0196600103023900 . jpa-00242725

\section{HAL Id: jpa-00242725 https://hal.science/jpa-00242725}

Submitted on 1 Jan 1966

HAL is a multi-disciplinary open access archive for the deposit and dissemination of scientific research documents, whether they are published or not. The documents may come from teaching and research institutions in France or abroad, or from public or private research centers.
L'archive ouverte pluridisciplinaire HAL, est destinée au dépôt et à la diffusion de documents scientifiques de niveau recherche, publiés ou non, émanant des établissements d'enseignement et de recherche français ou étrangers, des laboratoires publics ou privés. 


\title{
UTILISATION D ORDINATEURS \\ DANS L'ANALYSE DES SPECTRES DE MOLÉCULES DIATOMIQUES
}

\author{
Par Bernard GUERIN et .Jean D'INCAN, \\ Laboratoire de Spectroscopie et de Luminescence de l'Université de Lyon.
}

Résumé. - Un programme d'analyse des structures de rotation des spectres électroniques des molécules diatomiques a été établi dans le cas de transitions entre états de simplets dont les termes de rotation $F_{v}$ sont bien représentés par :

$$
F_{\mathbf{v}}(J)=B_{\nabla} J(J+1) \quad[1] .
$$

La méthode de tri exposée a été appliquée à l'aide d'un ordinateur IBM 1620. Elle peut se généraliser par l'établissement de formules théoriques adaptées aux structures plus complexes (doublets par exemple) et a été utilisée de manière plus complète avec un ordinateur du type IBM 7044.

Abstract. - A program for the analysis of the rotational structure of the electronic spectra of diatomic molecules has been established in the case of transitions between singlet states whose rotational terms $F_{\nabla}$ are well represented by :

$$
F_{\mathrm{v}}(J)=B_{\mathrm{v}} J(J+1) \quad[1] .
$$

The method of selection described has been applied by means of an IBM 1620 computer. It can be applied in general by establishing theoretical formula adapted to more complex structures (e. g. doublets) and has been used more completely with an IBM 7044 computer.

\section{Analyse des structures de rotation des spectres} électroniques des molécules diatomiques. - 1-1. Principes généraux. - La formule de définition des termes de rotation $F_{\mathrm{v}}(J)$ utilisée conduit à une représentation analytique des nombres d'ondes des composantes de la forme:

$$
\nu(m)=a+b m+c m^{2}[1]
$$

avec $m=-J, J, J+1$ suivant que les raies appartiennent à une branche $P, Q$ ou $R$.

Si l'on utilise les notations :

et

$$
\Delta v(m)=\nu(m \pm 1)-v(m)
$$

$$
\Delta^{2} \nu(m)=\Delta v(m \pm 1)-\Delta v(m)
$$

les signes + ou - se rapportant aux branches $R(+)$, $Q(+)$ et $P(-)$, la forme parabolique de $v(m)$ fait apparaître l'invariance de l'écart second

$$
2 C=2\left(B^{\prime}-B^{\prime \prime}\right) \text {. }
$$

La construction adoptée pour calculer des valeurs $\bar{v}_{i}$ théoriques, est alors :

où

$$
\overline{v_{i}}=\left(\nu_{i-1}\right)+\Delta v_{i-2}+\Delta^{2} v_{i-3}
$$

$$
\Delta^{2} \nu_{i-3}=\text { constante }=2\left(B^{\prime}-B^{\prime \prime}\right) .
$$

L'établissement d'une formule de moyenne sur l'ensemble des (vî) [2] conduit à la valeur

$$
\left(B^{\prime}-B^{\prime \prime}\right) \text { moyen }=\frac{\Delta v_{n-1}-\Delta \nu_{1}}{2(n-2)} .
$$

Le nombre d'ondes de la raie $\left(\nu_{n+1}\right)$ d'une branche peut donc être calculé au moyen de la formule de récurrence :

$$
\overline{\nu_{n+1}}=\left(\nu_{n}\right)+\Delta \nu_{n+1}+\frac{\Delta \nu_{n-1}-\Delta \nu_{1}}{n-2} .
$$

On obtient ainsi le principe d'une méthode générale. A partir des nombres d'ondes de $n$ raies successives d'une branche (définissant respectivement $n-1$ et $n-2$ écarts premiers et seconds) on peut calculer les valeurs théoriques $\overline{\nu_{n+1}}$ qui permettent de détermirer les $\left(\nu_{n+1}\right)$ par comparaison aux données expérimentales, dans un domaine d'incertitude dépendant de la précision des mesures.

Si $\delta \nu$ est l'incertitude expérimentale, on peut affirmer qu'une raie $\nu_{n+1}$ peut faire partie de la branche étudiée si l'irégalité (2) est vérifiée [2] :

$$
\left|\left(\nu_{n+1}\right)-\overline{\nu_{n+1}}\right| \leqslant \tau(n), \text { où } \tau(n)=4 \frac{n-1}{n-2} \delta \text {. }
$$

La méthode d'analyse est basée sur ce résultat et tient compte du fait que l'inégalité (2) est nécessaire mais non suffisante. L'intervalle $\tau(n)$ tend vers $4 \delta v$ quand $n$ augmente. Il est done nécessaire d'améliorer le processus de détermination des valeurs théoriques pour diminuer $\tau(n)$ plus rapidement. Des formules de lissage peuvent ètre utilisées pour améliorer les calculs théoriques effectués par la méthode précédente. La méthode des 
moindres carrés conduit aux valeurs théoriques $\lceil 2,3\rceil$ :

$$
v_{n+1}=\frac{3}{2 m\left(2 m^{2}-3 m+1\right)}
$$

$\sum_{k=1}^{2 m}\left[6 m^{2}+3 m+1-3 k(4 m+1)+5 k^{2}\right]\left(\nu_{n-k}\right)$.

La valeur $2 m+1$ indique le nombre de données expérimentales à partir desquelles s'effectue le calcul théorique de $\overline{\nu_{n+1}}$. L'intervalle $\tau(n)$ associé à cette méthode de lissage est $\leqslant 4 \delta \nu$ pour $n \geqslant 8$. Le programme de tri peut alors être divisé en deux parties. On détermine d'abord huit raies d'une série en calculant les valeurs théoriques des nombres d'ordes par réajustement de l'écart second et l'on utilise ensuite les formules de lissage pour attribuer les composantes suivantes (fig. 1). Ces techniques de calcul permettent d'extraire des séries de raies de type parabolique à partir de l'ensemble des $N$ raies expérimentales étudiées.

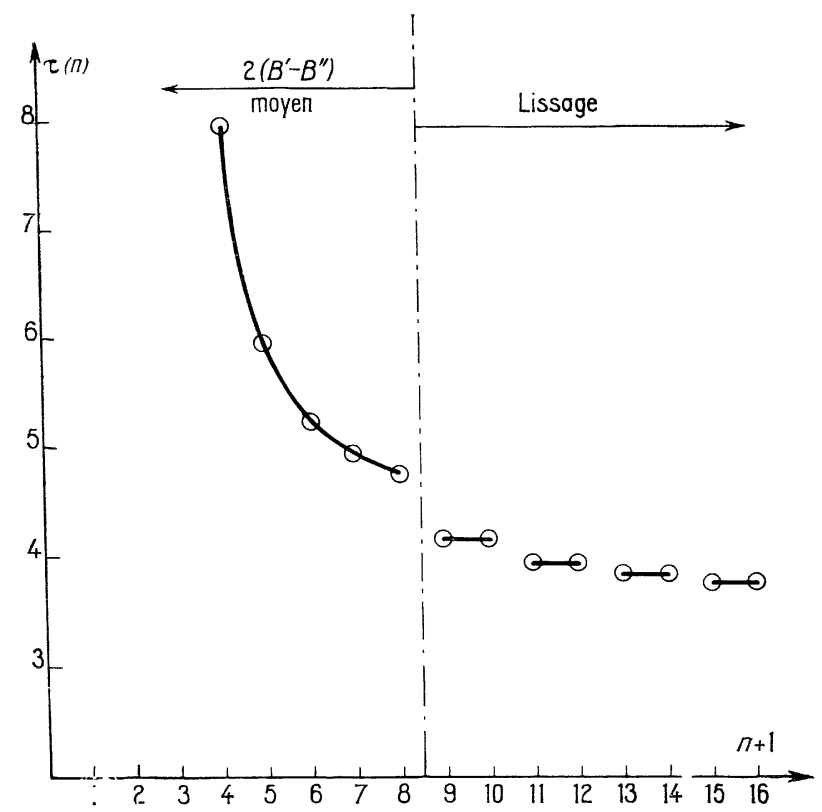

Fig. 1.

Pour classer les résultats, il est nécessaire de regrouper les branches appartenant à une même bande. Il suffit pour cela de remarquer que le nombre d'ondes de l'arête d'une branche est indépendant des valeurs attribuées aux nombres quantiques $m$ des différentes composantes de rotation (fig. 2).

Si $\left(\nu_{H}\right)_{R, P}$ et $\left(\nu_{H}\right)_{Q}$ sont les nombres des arêtes des branches $R(m)$ et $P(m)$ d'une part, et $Q(m)$ d'autre part, le calcul des $\nu_{H}$ permet de regrouper les branches appartenant à une même bande. Le calcul théorique des nombres d'ondes s'effectue à

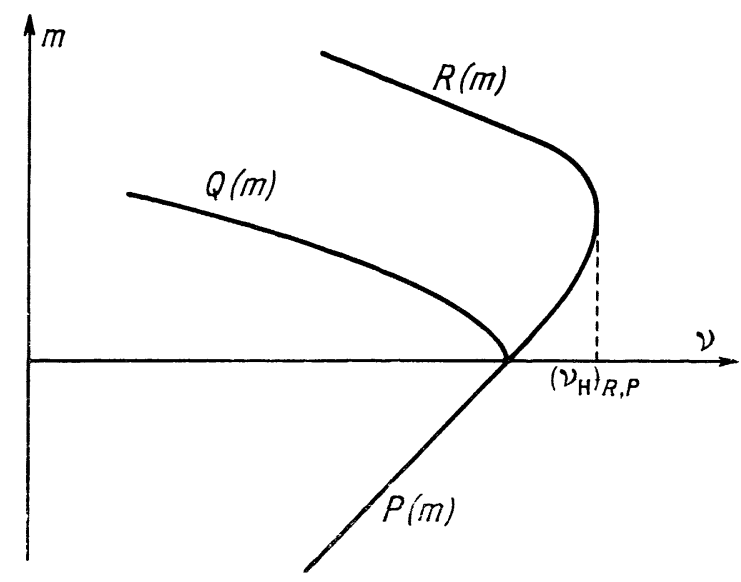

FIG. 2.

partir de la relation (1) où $a$ et $b$ sont inconnus (seul l'écart second $2 c$ est directement calculable). Une transformation analytique de cette expression en fonction des écarts premiers et seconds permet de déterminer des valeurs

$$
v_{\mathrm{H}}=a-\left(b^{2} / 4 c\right)[4] \text {. }
$$

Une formule d'interpolation de Newton [2] a été utilisée pour exprimer les valeurs $\overline{v(m)}$ des com. posantes d'une branche quelconque :

$$
\begin{aligned}
\overline{\nu(m)}=\nu\left(m_{0}\right) & +\frac{m-m_{0}}{h} \Delta v\left(m_{0}\right) \\
+ & \left(\frac{m-m_{0}}{h}\right)\left(\frac{m-m_{0}}{h}-1\right) \Delta^{2} v\left(m_{0}\right)
\end{aligned}
$$

où $h$ est un nombre entier algábrique,

$$
\begin{aligned}
& \Delta v\left(m_{0}\right)=v\left(m_{0}+h\right)-v\left(m_{0}\right) \\
& \Delta^{2} v\left(m_{0}\right)=v\left(m_{0}+2 h\right)-2 v\left(m_{0}+h\right)+v\left(m_{0}\right) . \\
& \nu\left(m_{0}\right) \text { et } v\left(m_{0}+2 h\right) \text { expriment les nombres }
\end{aligned}
$$
d'ondes de la première et de la dernière raie connue.

Cette nouvelle forme analytique développée suivant les puissances croissantes de $m$ conduit à une nouvelle expression de $\overline{\nu_{\text {H }}}[2]$

$\overline{\nu_{\mathrm{H}}}=\nu\left(m_{0}\right)+\frac{\Delta \nu\left(m_{0}\right)}{2}\left[1-\frac{\Delta \nu\left(m_{0}\right)}{\Delta^{2} \nu\left(m_{0}\right)}\right]-\frac{\Delta^{2} \nu\left(m_{0}\right)}{8}$.

On peut ainsi calculer $\overline{\nu_{\mathrm{H}}}$ toutes les fois qu'une branche a été déterminée par l'ordinateur, ce qui permet de regrouper éventuellement les séries.

Notons que l'expression $\Delta^{2} \nu\left(m_{0}\right) / 2 h^{2}$ exprime la valeur $\left(B^{\prime}-B^{\prime \prime}\right)$, ce qui permet souvent d'améliorer ce calcul de $\overline{\nu_{H}}$ si $\left(B^{\prime}-B^{\prime \prime}\right)$ a été déterminé d'une façon plus précise.

1-2. Réalisation du programme. - Pour effec- 
tuer le programme de tri, nous ne faisons intervenir que quatre données initiales :

- Écart premier maximum $T$.

- Écart second maximum (exprimé en valeur absolue).

- Nombre de raies $N$ à trier.

- Erreur expérimentale.

Ce procédé réduit au maximum le nombre de données, par rapport aux programmes déjà existants $[5]$.

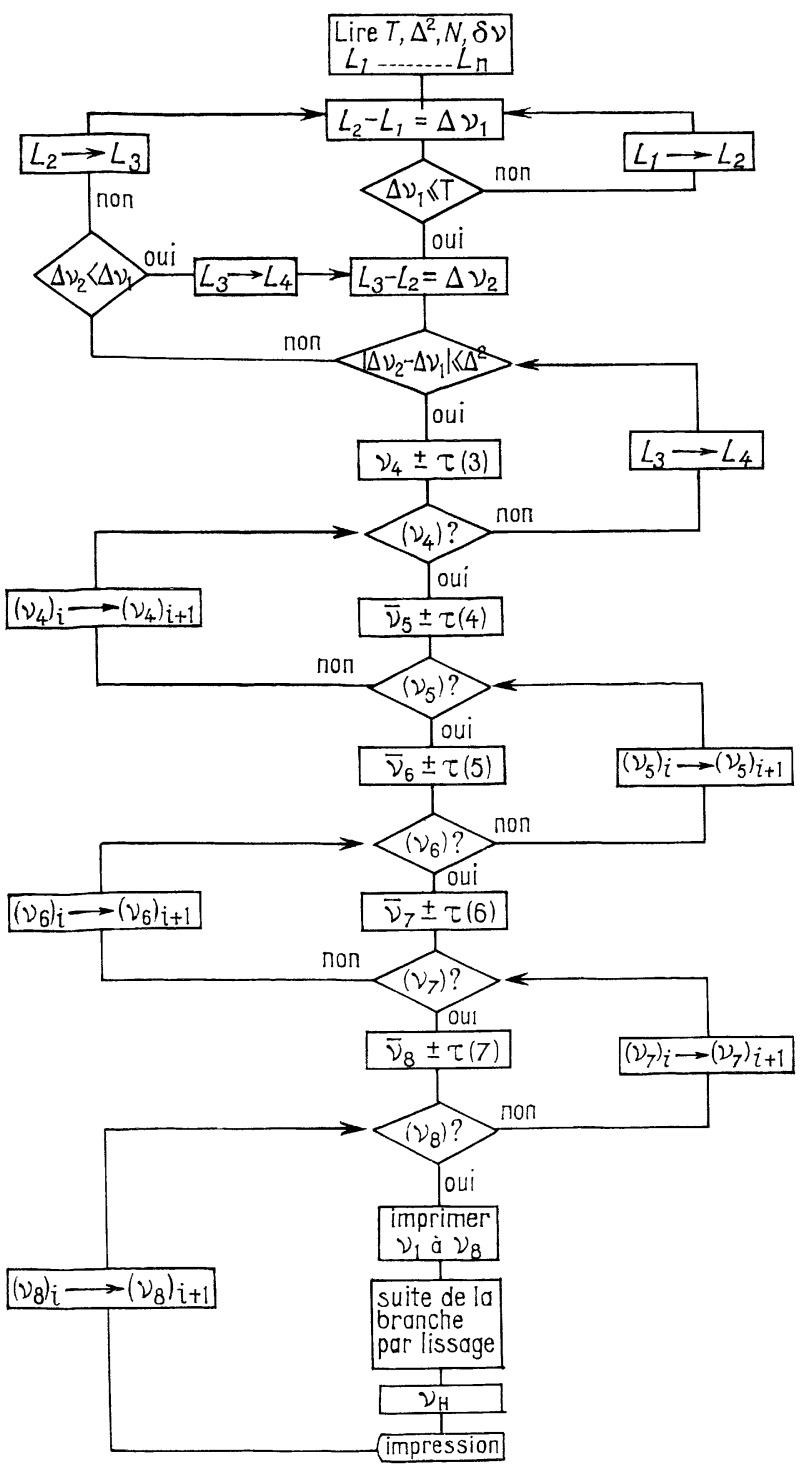

FIG. 3.

L'écart $T$ devra être élevé si le spectre est dégradé vers le rouge, et faible dans le cas contraire. L'écart $\Delta^{2}$ devra être choisi supérieur ou égal à la valeur réelle de $2\left|B^{\prime}-B^{\prime \prime}\right|$. L'organigramme récapitulatif est indiqué dans la figure 3 .
1-3. Résultats obtenus. - La méthode de classement des raies exposée a été testée sur la bande $(6,2)$ du système $\mathrm{D}^{2} \Pi_{\mathrm{g}} \rightarrow \mathrm{A}^{2} \Pi_{\mathrm{u}}$ de $\mathrm{N}_{2}^{+}[6]$.

Cette bande, dégradée vers le rouge, formée de quatre branches, contient 103 raies, certaines d'entre elles pouvant appartenir à des branches différentes.

Pour classer ces raies, nous avons utilisé :

$$
\begin{gathered}
N=103, \quad T=70 \mathrm{~cm}^{-1}, \\
\Delta^{2}=4 \mathrm{~cm}^{-1}, \quad \delta \nu=0,3 \mathrm{~cm}^{-1} .
\end{gathered}
$$

2. Détermination de la numérotation quantique. - 2-1. Détermination approghée Des NOMbres QUAN'TIQUES DE ROTATION A ATTRIBUER AUX COMPOSANTES D'UNe BRANChE. - La méthode, habituellement utilisée pour résoudre ce problème, consiste à fixer arbitrairement les valeurs des nombres quantiques $J$ des composantes d'une branche et à calculer les constantes $B^{\prime}+B^{\prime \prime}, B^{\prime}, B^{\prime \prime}$ par les formules de combinaison [7] :

$$
\begin{aligned}
& B^{\prime}=\frac{R(J)-P(J)}{4 J+2} \\
& B^{\prime \prime}=\frac{R(J-1)-P(J+1)}{4 J \perp 2} \\
& B^{\prime}+B^{\prime \prime}=\frac{R(J-1)-P(J)}{2 J} .
\end{aligned}
$$

Si l'on ne peut obtenir des résultats satisfaisants avec les valeurs de $J$ fixées, on change $J$ et l'on recommence les calculs jusqu'à l'obtention de valeurs constantes.

Les relations de combinaison (3), (4), (5) permettent d'écrire [2] :

$$
J=\frac{R(J-1)-P(J)}{2\left(B^{\prime}+B^{\prime \prime}\right)}
$$

avec

$$
B^{\prime}+B^{\prime \prime}=[\Delta R(J-1)-\Delta P(J)] / 2 \quad \text { (fig. 4). }
$$

Ce mode de calcul évite ainsi l'utilisation des valeurs de $J$. Pour une attribution $P(J), R(J)$ donnée, on calcule $B^{\prime}+B^{\prime \prime}$ et $J$ autant de fois qu'il existe de termes $[\Delta R(J-1)-\Delta P(J)]$.

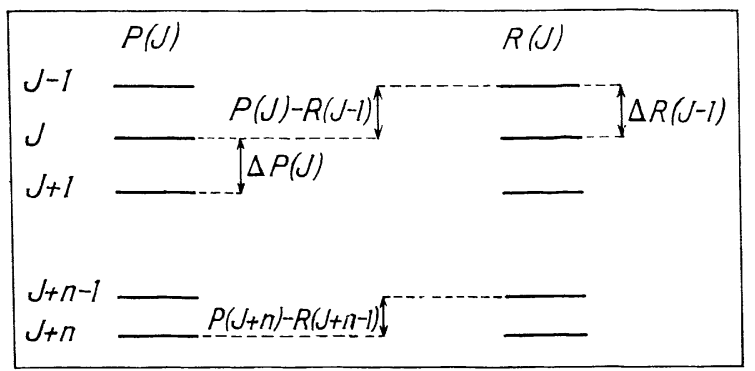

FIG. 4. 
Si les valeurs de $B^{\prime}+B^{\prime \prime}$ restent constantes, au terme d'erreur près, et que $J$ progresse en moyenne d'une unité quand on passe d'une raie à la suivante, la combinaison $[R(J), P(J)]$ est correcte, et l'on peut appliquer les formules de combinaisons classiques pour évaluer $B^{\prime}$ et $B^{\prime \prime}$ plus rigoureusement. Les nombres quantiques $J$ étant connus à une ou deux unités près, on peut préciser les différentes valeurs obtenues par des relations plus rigoureuses faisant intervenir des moyennes.

2-2. Calgul de la valeur moyenne de $B^{\prime}+B^{\prime \prime}$ ET DÉTERMINATION EXACTE DE LA NUMÉROTATION QUANTIQUE.

On peut écrire :

$$
2\left(B^{\prime}+B^{\prime \prime}\right)=\Delta R\left(J_{i}-1\right)-\Delta P\left(J_{\imath}\right) .
$$

Pour les différentes raies successives d'une bande, on a :

$$
\begin{array}{r}
2\left(B^{\prime}+B^{\prime \prime}\right)=-R(J+n-2)+R(J+n-1) \\
+P(J+n-1)-P(J+n) .
\end{array}
$$
[2] :

Ces $n$ valeurs de $2\left(B^{\prime}+B^{\prime \prime}\right)$ permettent d'écrire $\left(B^{\prime}+B^{\prime \prime}\right)_{\text {moyen }}=\{[P(J)-R(J-1)]$

soit

$$
-[P(J+n)-R(J+n-1)]\} / 2 n
$$

avec

$$
J=[R(J-1)-P(J)] / 2 B_{m},
$$

$$
B_{m}=\left(B^{\prime}+B^{\prime \prime}\right)_{\text {moyen }} \text {. }
$$

Si l'on tient compte du fait que les valeurs de $\boldsymbol{J}$ doivent progresser d'une unité d'une raie à la suivante, on obtient :

$$
J_{0}=\frac{R\left(J_{0}-1\right)-P\left(J_{0}\right)}{2 B_{m}}, \ldots
$$

$$
J_{n}=\frac{R\left(J_{0}+n-1\right)-P\left(J_{0}+n\right)}{2 B_{m}}=J_{n}+n
$$

soit

$$
J_{n}=\frac{\sum_{i=-1}^{n-1} R(J+i)-\sum_{i=0}^{n} P(J+i)}{\left.2 B_{m}{ }^{\prime} n+1\right)}+\frac{n}{2} .
$$

On peut ainsi obtenir sans ambiguïté la valeur du nombre quantique de rotation d'une raie.

3. Conclusion. - Les différentes relations que nous avons établies permettent de déterminer $J$ et $B^{\prime}+B^{\prime \prime}$.

Les étapes du programme sont donc les suivantes :

i) Analyse des structures de rotation ( $f$ ig. 3).

ii) Calcul des valeurs de $J$ et $B^{\prime}+B^{\prime \prime}$ à l'aide des relations (6) et (7) respectivement.

iii) Si l'analyse des résultats montre que $B^{\prime}+B^{\prime \prime}$ est constant au terme d'erreur $2 \delta \mathrm{V}$ près et que $J$ progresse d'une unité en moyenne on utilise alors les formules (8) et (9) pour améliorer la précision.

$\mathrm{Si}$ au contraire $\left(B^{\prime}+B^{\prime \prime}\right)$ varie dans un intervalle d'erreur supérieur à $2 \delta \nu$, on décale les raies $R(J)$ par rapport aux raies $P(J)$ jusqu'à l'obtention de résultats cohérents.

\section{Notations utilisées.}

$\overline{v_{i}}=$ valeur calculée.

$\left(v_{i}\right)=$ valeur expérimentale.

$\left(v_{i}\right)_{j}=$ ensemble des valeurs expérimentales comprises dans l'intervalle $\left[\bar{\nu}_{i} \pm \tau(i-1)\right]$.

$\left(v_{i}\right) ?=$ une valeur expérimentale existe-t-elle dans l'intervalle $\left[\overline{v_{i}} \pm \tau(i-1)\right]$ ?

$A_{i} \rightarrow A_{i+1}=$ remplacer $A_{i}$ par $A_{i+1}, A_{i+1}$ par $A_{i+2}$, etc...

Manuscrit reçu le 22 avril 1966.

\section{BIBLIOGRAPHIE}

[1] Herzberg (G.), Spectra of diatomic molecules, Van Nostrand Company, 1961, p. 169.

[2] Gú́rin (B.), Thèse 3e cycle, Lyon, 1965.

[3] Mineur (H.), Techniques de Calcul Numérique, Librairie Polytechnique, 1952, p. 453.
[4] Herzberg (G.), Loc. cit., p. 171.

[5] Phillips (J.), Astrophys. J., 1959, 130, 308.

[6] D'Incan (J.), Thèse de Doctorat ès Sciences, Lyon, 1959.

[7] Herzberg (G.), Loc. cit., p. 177. 